Discussion/conclusion Our project reflects Chlamydia as an important cause of PID in younger women. This supports the latest guidance recommending repeat Chlamydia screening in under 25 s to identify reinfections and reduce the risk of complications such as PID.

\section{P093 AUDIT ON THE MANAGEMENT OF EPIDIDYMO-ORCHITIS IN A LONDON-BASED LEVEL 3 SEXUAL HEALTH CLINIC}

Temi Lampejo* ${ }^{*}$ Mohamed Abdulcadir. Chelsea \& Westminster Hospital, London, UK

\subsection{6/sextrans-2016-052718.147}

Background/introduction The aetiology of epididymo-orchitis is largely related to a patient's age with sexually transmitted pathogens being the common aetiological agents in those under 35 years of age. In individuals aged over 35 uropathogens represent the commonest cause. National guidelines exist for the appropriate management of this condition.

Aim(s)/objectives To assess the management of epididymo-orchitis in our clinic with reference to the BASHH guidelines.

Methods A case note review of all men with epididymo-orchitis attending our clinic between January and June 2015. Age at time of diagnosis, investigations and treatment decisions were recorded.

Results A total of 59 patients were identified ranging from age 16 to 67 . Only $66 \%$ of patients had all four recommended microbiological investigations performed (target 90\%). Nineteen patients did not have an MSU microscopy/culture performed and 7 had no urethral smear. All patients were tested for chlamydia and gonorrhoea. All 59 patients were prescribed an appropriate antibiotic regimen. The 5 patients who did not respond clinically had a documented plan for further clinical action.

Abstract P093 Table 1 Epididymo-orchitis

\begin{tabular}{llll}
\hline & Age $\leq 35$ years & Age $>35$ years & Total \\
\hline No. of patients & 38 & 21 & 59 \\
CT positive & 5 & 0 & 5 \\
GC positive & 0 & 0 & 0 \\
MSU positive & 1 & 1 & 2 \\
\hline
\end{tabular}

Discussion/conclusions This audit demonstrated that patients attending our clinic were treated in concordance with national guidelines and the vast majority showed a good clinical response. However, lack of routine urine sampling for microscopy/culture was evident. Although a urine dipstick was performed in most cases, guidelines do stipulate that this only serves as a useful adjunct. As a result of this audit our department intends to obtain an MSU for culture in all cases of epididymo-orchitis.

\section{P094 CHLAMYDIA TRACHOMATIS (CT) POSITIVITY RATE AT 2 WEEK NEISSERIA GONORRHOEAE (NG) TEST OF CURE (TOC)}

Nicolo Girometti, Joseph Scott, Kimberly Rhodes, Alan McOwan*, Nneka Nwokolo, Gary Whitlock. ${ }^{56}$ Dean Street, Chelsea \& Westminster Hospital, London, UK
Background/introduction Whilst guidelines recommend NG TOC 2 weeks after treatment, there is little data on the optimum time to perform a TOC for CT in those for whom this is indicated. Current BASHH guidelines recommend deferring TOC for at least 3 weeks after treatment because residual chlamydial DNA may persist.

Aim(s)/objectives Patients who are treated for NG and CT coinfection re-attending for subsequent NG TOC are tested for both infections by NAAT providing the opportunity to evaluate the CT positivity rate at re-attendance.

Methods A retrospective case review of co-infected GC/CT positive (analysed with Cepheid GeneXpert) patients tested in a London sexual health clinic over 12 consecutive months was performed. TOC details were evaluated, and appropriate antibiotic treatment according to $\mathrm{BASHH}$ guidelines was assessed.

Results 480 patients tested positive for both infections and 132 attended for TOC within 21 days of treatment (median 15 days, IQR 14-17). Of these 131 were male, of whom 126 MSM; median age was $35 \mathrm{y}$ and median number of sexual partners in previous 3 months was 5. Site of CT infection was rectal (94), urethral (49), throat (11), vulvovaginal (1). At TOC, 6 (4.5\%) had a persistent positive CT NAAT: rectum (3), urethra (3). One patient with persistent rectal CT had received treatment with azithromycin; the other 5 received BASHH preferred treatment. By comparison, $3(2.3 \%)$ had a positive NG NAAT at TOC.

Discussion CT positivity 15 days after treatment is low, suggesting that TOC at 2 weeks may be a possible management strategy.

\section{P095 GETTING HIGH AND HAVING SEX- ARE YOUNG WOMEN JOINING THE PARTY?}

Nicky Dearing*, Sophie Herbert. Nottingham University Hospitals NHS Trust, Nottingham, UK

\subsection{6/sextrans-2016-052718.149}

Background Use of 'chems' by MSM (men who have sex with men) is reported widely and is associated with poor sexual health outcomes but less is known for the general GU clinic population.

Aims To determine the proportion of men and women reporting recreational drug use and identify sexual risk taking and health outcomes.

Methods Patients attending GUM from 1-21 ${ }^{\text {st }}$ December 2015 were invited to complete an anonymous paper questionnaire. Age, sexual orientation, sexual partners, STIs, smoking, drug and alcohol use were collected.

Results 128 men (32.8\% MSM) and 101 women responded. 19\% women, 36\% heterosexual men (HM) and 52\% MSM reported recreational drug use in the past 12 months. Women users were younger (age range 19-42, median 23) and their preferred drug was Cocaine (12\%). Men were older (age range 1967, median 28), cocaine was a preferred drug (28\% HM, 19\% MSM) but MSM also used Mephadrone, Ecstasy and Viagra equally (19\%). Users reported UPSI with multiple partners in the last 3 months more often (68\% MSM, 50\% HM, 53\% females) compared with non users (30\% MSM, 26\% HM and 17\% females). Female users reported the highest recent STI rates, 68\% (MSM 55\%) and non-consensual sex (21\%).

Discussion We found significant drug use and risky sexual behaviour amongst heterosexuals, although MSM remain the highest 